\title{
NEW MAGNETIC RESONANCE SPECTROSCOPY BIOMARKER FOR MONITORING NEURODEGENERATIVE DISEASES: ANIMAL MODELS
}

\author{
Svatava Kašparováa, Zuzana Sumbalováb, Jaromír Horeckýc, Peter Bystrickýa, \\ Vladimír Mlynárik $^{\mathrm{d}}$, Anna Gvozdjákováb, Tibor Liptaja
}

\author{
a NMR Laboratory, Faculty of Chemical and Food Technology, Slovak University of Technology in Bratislava \\ ${ }^{b}$ Pharmacobiochemical Laboratory of the $3^{r d}$ Department of Internal Medicine, Faculty of Medicine, Comenius University, \\ Bratislava, Slovakia \\ c Slovak Medical University Bratislava, Slovakia \\ d Metabolic Imaging, EPFL, Lausanne, Switzerland
}

Received: June 10, 2005; Accepted: September 25, 2005

Key words: Creatine kinase/Saturation transfer 31P NMR/Chronic cerebral hypoperfusion/Neurodegenerative diseases/ Animal model

Creatine kinase (CK) plays a central role in energy transfer in cells with high-energy demands, and the enzyme is rather susceptible to oxidative inactivation. The aim of the present study was to investigate whether the rate constant of forward CK reaction $\left(k_{\mathrm{for}}\right)$ is a suitable indicator of alterations in cerebral energy metabolism. We monitored $k_{\text {for }}$ in the rat brain non-invasively by in vivo phosphorus $\left({ }^{31} \mathrm{P}\right)$ magnetic resonance spectroscopy (MRS). To alter energy metabolism, we applied following experimental models: Huntington's disease, diabetes mellitus, chronic alcohol intoxication and chronic cerebral hypoperfusion (vascular dementia model). Results of our ${ }^{31} \mathrm{P}$ MRS experiment confirm importance of creatine kinase/phosphocreatinine $(\mathrm{CK} / \mathrm{PCr})$ system in the regulation of brain energy metabolism in vivo because a kinetic parameter $k_{\text {for }}$ was significantly changed in all above animal models that simulate neurodegenerative diseases or commonly during oxidative stress. Using this method we distinguished vascular dementia (VD) and Huntington disease (HD), because in VD model a kinetic parameter $k$ decreased and in the case HD increased. Considering the importance of CK for the maintenance of energy homeorstasis in the brain, it is conceivable that an alteration of this enzyme activity in the brain may be one of the mechanisms by which various neurodegenerative diseases might be monitored just by means saturation transfer method ${ }^{31} \mathrm{P}$ MRS.

\section{INTRODUCTION}

Metabolism of adenosine triphosphate (ATP) is key process for cell live and involves ATP synthesis and its utilization for supporting brain function and activation. The ATP metabolism is tightly coupled to the phosphocreatinine (PCr) metabolism via the enzyme system of creatine kinase. CK plays a central role in energy transfer in cells with high and fluctuating energy requirements and it is very susceptible to oxidative stress ${ }^{1-4}$. It would be valuable to establish a noninvasive approach for studying this chemical process in the animal (human) brain in vivo. Rate constants of $\mathrm{CK}$ reaction can be investigated by magnetization transfer in vivo ${ }^{31} \mathrm{P} \mathrm{MRS}$ experiment ${ }^{5,6}$. By means of this technique we studied reaction kinetics of reversible exchange of the phosphate group in the reaction catalyzed by CK

$$
\mathrm{PCr}^{2-}+\mathrm{MgADP}^{-}+\mathrm{H}^{+} \Leftrightarrow \mathrm{MgATP}^{2-}+\mathrm{Cr}
$$

in the adult and aged rat brains in various chronic models of neurodegenerative diseases ${ }^{7-11}$.
The forward rate constant of $\mathrm{CK}, k_{\text {for }}(\mathrm{PCr}=>\mathrm{ATP})$ was studied in adult and aged rat brains in following models of diseases: i) Huntington's disease (HD), ii) diabetes mellitus (DM), iii) alcohol intoxication and iv) vascular form dementia (VD) of Alzheimer's disease modeled by chronic cerebral hypoperfusion. The aim of these experiments was to demonstrate that in chronic pathological states the pseudo first-order rate constant $k_{\text {for }}$ of the CK forward reaction is a better indicator of changes in the brain energy metabolism than the conventional steadystate in vivo ${ }^{31} \mathrm{P} \mathrm{MRS}$.

\section{MATERIAL AND METHODS}

\section{Animal models}

Wistar rats with the age 3-10 (adult) and 14-24 (aged) month were used in ${ }^{31} \mathrm{P}$ MRS experiments.

Huntington's disease (HD) can be simulated in an animal model with systemic administration of 3-nitropropionic acid (3-NP). Male Wistar rats with the age 2024 months were injected i.p. with $3-\mathrm{NP}$ ( $10 \mathrm{mg} / \mathrm{kg} /$ every 
$12 \mathrm{~h}$ ) for 11 days to develop chronic model of HD $\left(\right.$ ref. $\left.^{2}\right)$. Group $\mathrm{QE}+\mathrm{HD}$ received coenzyme $\mathrm{Q}_{10}\left(\mathrm{CoQ}_{10}\right)$ and vita$\min \mathrm{E}\left(250 \mathrm{mg} \mathrm{CoQ}{ }_{10}+530 \mathrm{mg}\right.$ vit.E/ $/ \mathrm{kg} /$ day, i.g. $)$ during 10 days before application of 3-NP $\left(\right.$ ref. $\left.^{10}\right)$.

Diabetes mellitus (DM) was induced in 11 months old male Wistar rats by single dose of streptozotocin (STZ) $(55 \mathrm{mg} / \mathrm{kg}$, i.v.). The animals were kept without insulin for 3 months. Group $\mathrm{DM}+\mathrm{Q}$ received $\mathrm{CoQ}_{10}(20 \mathrm{mg} / \mathrm{kg} /$ day, i.p.) during 3 months, starting with the onset of DM $\left(\right.$ ref. ${ }^{11}$ ).

Chronic alcohol intoxication was modeled by administration of $30 \%$ ethanol ( $3 \mathrm{ml} /$ day, intragastrically) to 3 months old female rats for the period of 3 weeks. Control group (C) received water as a vehicle ${ }^{7,8}$.

Vascular dementia (VD) model was prepared by a minimally invasive surgical technique of 3-vascular occlusion by brachiocephalic trunk, which eliminated simultaneously right common carotid artery and right vertebral artery ${ }^{9,12}$. 6-10 months old (adult) and 14-17 months old (aged) male Wistar rats were used in this experiment. Saturation transfer in vivo ${ }^{31} \mathrm{P}$ MRS were performed two and ten weeks after vascular occlusion ${ }^{8,9}$.

\section{In vivo ${ }^{3 l} \mathrm{P}$ magnetic resonance spectroscopy}

In vivo ${ }^{31} \mathrm{P}$ MRS experiments were performed at $4.7 \mathrm{~T}$ on SISCO 200/300 imaging spectrometer equipped with horizontal bore magnet for measurements on animals. ${ }^{31} \mathrm{P}$ MR spectra were collected using $16 \mathrm{~mm}$ surface coil. The static magnetic field was shimmed using the proton signal of water that showed a typical line width of $20-35 \mathrm{~Hz}$. Relative concentrations of phosphate metabolites were determined from integrals of their signals in ${ }^{31} \mathrm{P}$ MR spectra using program MESTRE-C 1.5.1. Time-dependent ${ }^{31} \mathrm{P}$ MRS saturation transfer was applied to determine the pseudo-first order rate constant of forward CK reaction $\left(k_{\text {for }}\right)$ as described previously $y^{6,9,13,14}$. Time dependent saturation transfer allows one to measure simultaneously two parameters, $\mathrm{T}_{1}$ and $k_{\text {for }}$. Saturation of the $\gamma$-ATP resonance for increasing time periods induces an exponential decay of the PCr resonance to a new steady state (Fig. 1).

The saturation was accomplished by the DANTE pulse sequence. The $\mathrm{k}_{\text {for }}$ was calculated according to the equation $^{9,13,14}$

$$
k_{f o r}=\left[1-M_{(\mathrm{PCr})}^{*} / M_{0(\mathrm{PCr})}\right] / T_{\text {lapp(PCr) }}
$$

where $M_{\mathrm{O}(\mathrm{PCr})}$ and $M^{*}{ }_{(\mathrm{PCr})}$ are the $\mathrm{PCr}$ signal intensities in the reference spectrum and in the spectrum after 10second irradiation of the $\gamma$-ATP resonance, respectively. $T_{\text {lapp(PCr) }}$ is the apparent longitudinal relaxation time of the PCr signal in the presence of the $\gamma$-ATP saturation. To check the validity of the results, the $T_{1 \mathrm{PCr}}$ values were calculated using the following equation ${ }^{9,13,14}$

$$
T_{1 \mathrm{PCr}}=T_{1 \mathrm{sPCr}}\left[M_{\mathrm{PCr}}^{0} / M_{\mathrm{PCr}}^{\infty}\right]
$$

Calculated values $k_{\text {for }}$ and $T_{1 \mathrm{PCr}}$ were expressed as means \pm standard deviations. The Student's $t$ tests were performed to compare differences between groups.

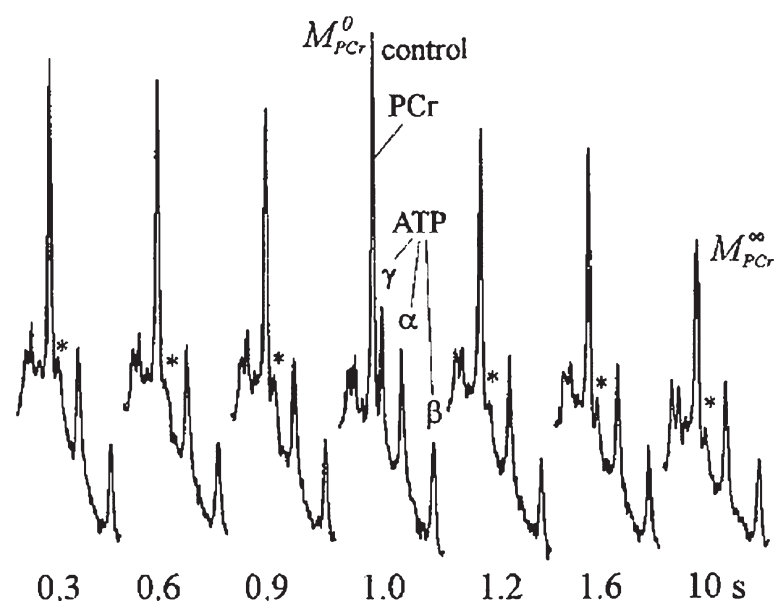

Fig. 1. Time dependent ${ }^{31} \mathrm{P}$ NMR saturation transfer experiments. Series of saturation experiments with variable time of irradiation (0.3-10 s) of $\gamma$ ATP signal. The length of the saturation period is given below each spectrum. In the control experiment, the saturation was off-resonance. The saturated $\gamma$ ATP signal is labelled with asterisk.

\section{RESULTS AND DISCUSSION}

Our findings suggest that the CK reaction could play a key role in energetic system of adult and aged brains in above models of neurodegenerative diseases. While the analysis of steady-state ${ }^{31} \mathrm{P}$ MR spectra revealed significant alteration in brain $\mathrm{PCr} / \beta \mathrm{ATP}$ ratio only in diabetic group, we found significant changes of brain $k_{\text {for }}$ in all chronic models of neurodegenerative diseases. Compared to controls $k_{\text {for }}$ increased in HD and DM models and decreased in chronic alcohol intoxication and VD models (Table 1).

From kinetic ${ }^{31} \mathrm{P}$ MRS spectra we found in case of HD small (+13.4 \%), but statistically significant an increase in $k_{\text {for }}$ (Table 1). Exact mechanism responsible for the increase of the pseudo-first-order rate constant for the CK reaction $\mathrm{k}_{\text {for }}$ is presently not clear. However, in vivo ${ }^{31} \mathrm{P}$ MRS transfer measurements revealed a correlation between CK flux and brain 2-deoxyglucose uptake ${ }^{5,6}$. Our result of increased activity of CK corresponds well with reported increased 2-deoxyglucose uptake in basal ganglia of HD patients ${ }^{1,5}$.

Similar increase of $k_{\text {for }}(+22.9 \%)$ we obtained in the model of diabetes mellitus in which also increased brain $\mathrm{PCr} / \beta$ ATP ratio was found (Table 1 ). In both above mentioned models the function of respiratory chain in brain mitochondria was diminished ${ }^{11}$. We suppose that decreased brain mitochondrial ATP production could be partly compensated in a certain stage of these pathological states by increased activity of CK system. The fact that the increase of $k_{\text {for }}$ in these models of neurodegenerative diseases could be prevented by application of $\mathrm{CoQ}_{10}$ provides evidence that $k_{\text {for }}$ reflecting activity of $\mathrm{CK}$ system is sensitive marker of changes in brain energy metabolism ${ }^{10,11}$. 
Table 1. Parameters determined by ${ }^{31} \mathrm{P}$ MRS methods in the rat brain in models of neurodegenerative diseases

\begin{tabular}{|c|c|c|c|c|}
\hline Model of neurodegenerative disease & n & $k_{\text {for }}\left(\mathrm{s}^{-1}\right)$ & $T_{1}(\mathrm{PCr})(\mathrm{s})$ & PCr/ßATP \\
\hline \multicolumn{5}{|l|}{ 1. Huntington's disease } \\
\hline Control aged rats (20-24 months) & 6 & $0.304 \pm 0.012$ & $3.079 \pm 0.076$ & $2.032 \pm 0.128$ \\
\hline Huntington disease (HD) model & 5 & $0.350 \pm 0.015 *$ & $2.561 \pm 0.119$ & $2.164 \pm 0.145$ \\
\hline CoQ10+E + HD model & 4 & $0.313 \pm 0.012$ & $3.006 \pm 0.109$ & $2.212 \pm 0.117$ \\
\hline \multicolumn{5}{|l|}{ 2. Diabetes mellitus } \\
\hline Control aged rats (11 months) & 5 & $0.314 \pm 0.007$ & $2.702 \pm 0.229$ & $2.033 \pm 0.23$ \\
\hline Diabetes mellitus & 4 & $0.386 \pm 0.004 * * *$ & $3.105 \pm 0.127$ & $2.706 \pm 0.11^{* *}$ \\
\hline Diabetes melitus + CoQ10 & 4 & $0.310 \pm 0.013 \# \#$ & $3.028 \pm 0.123$ & $2.465 \pm 0.132$ \\
\hline \multicolumn{5}{|l|}{ 3. Chronic alcohol intoxication } \\
\hline $\mathrm{C}$ (female) & 5 & $0.377 \pm 0.02$ & $3.233 \pm 0.179$ & $2.641 \pm 0.092$ \\
\hline Alcohol & 4 & $0.307 \pm 0.01 *$ & $3.268 \pm 0.089$ & $2.553 \pm 0.042$ \\
\hline \multicolumn{5}{|l|}{$\begin{array}{l}\text { 4. VD model of Alzheimer disease } \\
\text { Adult rats }\end{array}$} \\
\hline Control adult rats (6-10 months) & 10 & $0.345 \pm 0.025$ & $3.521 \pm 0.256$ & - \\
\hline Vascular dementia model 2 weeks & 10 & $0.284 \pm 0.041$ & $3.227 \pm 0.239$ & - \\
\hline $\begin{array}{l}\text { Vascular dementia model } 10 \text { weeks } \\
\text { Aged rats }\end{array}$ & 6 & $0.262 \pm 0.042 *$ & $3.032 \pm 0.411$ & - \\
\hline Control aged rats (14-17months) & 8 & $0.301 \pm 0.013 a$ & $3.421 \pm 0.353$ & - \\
\hline Vascular dementia model 2 weeks & 6 & $0.213 \pm 0.042 * *$ & $3.501 \pm 0.416$ & - \\
\hline Vascular dementia model 10 weeks & 6 & $0.201 \pm 0.033 * *$ & $3.610 \pm 0.142$ & - \\
\hline
\end{tabular}

$\mathrm{n}$ - number of animals per group; $k_{\text {for }}$ - the pseudo-first order rate constant of forward CK reaction determined in brain in vivo by ${ }^{31} \mathrm{P}$ MRS saturation transfer; $T_{1}(\mathrm{PCr})$ - longitudinal relaxation time of phosphocreatine in absent $\gamma$-ATP saturation, a control parameter; $\mathrm{PCr} / \beta \mathrm{ATP}$ ratio calculated from in vivo ${ }^{31} \mathrm{P} \mathrm{MR}$ spectra of the rat brain. $\mathrm{C}-\mathrm{control}$ rats; ${ }^{*} p<0.05,{ }^{* *} p<0.01$ vs. control group, ${ }^{* \#} P<0.001$ vs. DM group, ${ }^{\text {a }} P<0.05$ vs. control adult rats

Long-term consumption of alcohol can lead to brain atrophy and neurological dysfunction. Ethanol and its metabolite acetaldehyde are directly neurotoxic and its acute and chronic intoxication with ethanol induces oxidative stress in CNS and peripheral nerves ${ }^{7}$. The conventional ${ }^{31} \mathrm{P}$ MRS revealed statistically significant decrease of intracellular $\mathrm{pH}$ in the brain of rats treated by ethanol, but it did not show any significant variation in the content of high-energy phosphates after 14 weeks of drinking the high ethanol doses ${ }^{7}$. However, measurement of fluxes in the direction from PCr to ATP CK reaction showed significant decrease of the forward $\mathrm{CK}$ rate constant in alcoholic rat brain ${ }^{2,8}$ (Table 1).

The study of cerebral hypoperfusion in rats is great importance for a better understanding of cerebrovascular dynamics in various neurodegenerative diseases including Alzheimer's disease (AD). The characteristic pathology of $\mathrm{AD}$ involves microvascular degeneration and chronic cerebrovascular insufficiency ${ }^{12}$. Two factors must be present before cognitive dysfunction and neurodegeneration is expressed in AD brain: advanced aging and presence of a condition that lowers cerebral perfusion ${ }^{12}$. Compared to the control group of healthy aged rats the hypoperfused rats showed statistically significant decrease in $k_{\text {for }}$ both 2 and 10 weeks after occlusion (Table 1 ). It should be noted here that adult and aged rats kept for 10 weeks under conditions of severe hypoperfusion, showed no statistically significant changes in conventional in vivo ${ }^{31} \mathrm{P}$ MRS spectrum. Dysfunction of the CK system under AD conditions has also recently been reported ${ }^{4,15}$. The causes of decreased levels of cytosolic brain $\mathrm{CK}$ isoform (BB-CK) in postmortem brain in mental pathology are subject of discussions ${ }^{15}$. As it was recently demonstrated, BB-CK energy transfer in the brain is important for habituation and spatial learning behaviour ${ }^{16}$. Dynamic study of ${ }^{31} \mathrm{P}$ MR spectroscopy revealed strong reduction of $\mathrm{PCr}$ 
=>ATP phosphorus flux in mice with ablation of cytosolic BB-CK, while ATP and PCr levels were unaffected in these mice ${ }^{16}$. Thus, the rate constant of $\mathrm{CK}, k_{\text {for }}$ measured in the brain reflects changes of BB-CK activity ${ }^{8,9,16}$.

We confirmed that a metabolic capacity of the creatine kinase reaction in the brain in vivo was significantly changed in various animal models simulating neurodegenerative diseases, such as $\mathrm{AD}, \mathrm{HD}$ or generally during oxidative stress.

We have concluded that the investigation of the kinetic parameters using in vivo ${ }^{31} \mathrm{P}$ MRS magnetization transfer method contributed to a better understanding of the underlying processes in various neurological disorders. Using this method we distinguished $\mathrm{AD}$ and $\mathrm{HD}$, because $k_{\text {for }}$ decreased in AD model while it increased in HD. This technique can be used as a noninvasive in vivo biomarker for age-related neurodegenerative diseases as it can reveal energy metabolism impairment of brain tissue, which is not yet detectable by conventional MRS methods.

\section{ACKNOWLEDGEMENT}

This work was facilitated by the support of the Slovak State Program of Research and Development No. 2003SP200280203, and grants VEGA No. 1/4112/97-99, $1 / 7547 / 20,1 / 0546 / 03$.

\section{REFERENCES}

1. Beal M F. (1998) Mitochondrial dysfunction in neurodegenerative diseases. Biochim Biophys Acta 1366, 211-23.

2. Matthews RT, Yang L, Jenkins BG, Ferrante RJ, Rosen BR, Kaddurah Daouk R, Beal MF. (1998) Neuroprotective effects of creatine and cyclocreatine in animal models of Huntington's disease. J Neurosci 18,156-63.

3. McCord JM, Russel VJ. Inactivation of creatine phosphokinase by superoxide during reperfusion injury. In: Simic KA, Word JF, Von Sonntag C, editors. Oxygen Radicals in Biology and Medicine. New York: Plenum Press, 1988. p 869-73.

4. Yatin S M, Askenov M, Butterfield DA. (1999) The antioxidant vitamin $\mathrm{E}$ modulates amyloid $\beta$-peptide-induced creatine kinase activity inhibition and increased protein oxidation: Implications for the free radical hypothesis of Alzheimer's disease. Neurochem Res 24, 427-35.

5. Corbett RJT, Laptook AR. (1994) Age-related changes in swine brain creatine kinase catalyzed 31P exchange measured in vivo using 31P NMR magnetization transfer. J Cereb Blood Flow Metab 14, 1070-7.

6. Sauter A, Rudin M. (1993) Determination of creatine kinase kinetic parameters in rat brain by NMR magnetization transfer. J Biol Chem 682, 13166-71.

7. Braunová Z, Kašparová S, Mlynárik V, Mierisová Š, Liptaj T, Tkáč I, Gvozdjáková A. (2000) Metabolic changes in rat brain after prolonged ethanol consumption measured by $1 \mathrm{H}$ and 31P MRS experiments. Cell Mol Neurobiol 20, 703-15.

8. Kašparová S, Dobrota D, Mlynárik V, Pham TN, Liptaj T, Horecký J, Braunová Z, Gvozdjáková A. (2000) A Study of creatine kinase reaction in rat brain under chronic pathological conditions - chronic ischemia and ethanol intoxication. Brain Res Bull 53, 431-5.

9. Kašparová S, Brezová V, Valko M, Horecký J, Mlynárik V, Liptaj T, Vančová O, Uličná O, Dobrota D. (2005) Study of the oxidative stress in a rat model of chronic brain hypoperfusion. Neurochem Inter 46, 601-11.

10. Sumbalová Z, Kašparová S, Bystrický P, Kucharská J, Mlynárik V, Gvozdjáková A. (2002) Effect of coenzyme Q10 and vitamin E on brain and skeletal muscle energy metabolism in animal model of Huntington's disease. In: Third Conference of the International Coenzyme Q10 Association. London. p. 193-5.

11. Sumbalová Z, Kucharská, J, Kašparová S, Mlynárik V, Bystrický P, Uličná O, Vančová O, Singh RB, Gvozdjáková A. (2005) Brain energy metabolism in experimental chronic diabetes: long-term effect of coenzyme Q10 and $\omega-3$ fatty acids. Biologia, Bratislava, Suppl 11, p. ///.

12. de la Torre JC, Fortin T. (1994) A chronic physiological model of dementia. Behav Brain Res 63, 35-40.

13. Clark JF, Harris GI, Dillon PF. (1991) Multisite saturation transfer using DANTE and continuous wave. Magn Reson Med 17, 274-8.

14. Mlynárik V, Kašparová S, Liptaj T, Dobrota D, Horecký J, Belan V. (1998) Creatine kinase reaction rates in rat brain during chronic ischemia. Magnetic resonance materials in physics, biology, and medicine, MAGMA 7, 62-165.

15. Burbaeva GSh, Savushkina OK, Dmitriev AD. (1999) Brain isoforms of creatine kinase in health and mental diseases Alzheimer's disease and schizophrenia. Vestn Ross Akad Med Nauk (1), 2024.

16. Jost CR, Van der Zee CEEM, in't Zandt H J A, Oerlemans F, Verheij M, Streijger F, Fransen J, Heerschap A, Cools AR, Wieringa B. (2002). Creatine kinase B-driven energy transfer in the brain is important for habituation, and spatial learning behaviour, mossy fibre field size and determination of seizure susceptibility. Europ J Neurosci 15, 1692-1706. 